Poster presented at the 2K1BC Workshop

Experimental Cosmology at Millimetre Wavelengths

July 9-12, 2001, Breuil-Cervinia (Italy)

\title{
Sunyaev-Zel'dovich Effect and Morphology of Galaxy Clusters
}

\author{
R. Piffaretti*, Ph. Jetzer ${ }^{\dagger}$, D. Puy*, S. Schindler ${ }^{\ddagger}$ \\ ${ }^{*}$ Paul Scherrer Institute \\ and Institute of Theoretical Physics, University of Zürich - CH \\ ${ }^{\dagger}$ Institute of Theoretical Physics, University of Zürich \\ and Institute for Theoretical Physics, ETH Zürich - CH \\ ${ }^{\ddagger}$ Astrophysics Research Institute, Liverpool John Moores University - UK
}

\begin{abstract}
We investigate the influence of the finite extension and the aspherical geometry of a galaxy cluster on the estimate of the Hubble constant through the Sunyaev-Zel'dovich (SZ) effect. An analysis of a recent Chandra image of the galaxy cluster RBS797 indicates a strong ellipticity and thus a pronounced aspherical geometry. We estimate the total mass of RBS797 assuming spherical or ellipsoidal geometry and show that in the latter case the mass is about 10-17\% less than the one inferred for a spherical shape.
\end{abstract}

\section{Introduction}

The recent technical developments of millimetre receivers open new perspectives for more accurate measurements of the SZ effect and will thus trigger new developments on theoretical work (see the invited paper of Y. Rephaeli in this conference). Accurate measurements of the SZ effect are still difficult as well as their correct interpretation, indeed systematic errors can be significant. For example Cooray [1] showed the influence of projection effects and Sulkanen [2] pointed out that the shape of galaxy clusters could produce systematic errors. More recently, Puy et al. [3] investigated the SZ effect and the $X$-ray surface brightness for galaxy clusters with a non-spherical mass distribution.

In the first part of this communication we review the "classical" systematic errors such as cluster extension and geometry, by discussing the recent millimetre measurements of Mauskopf et al. [4] of the SZ effect in Abell 1835. In the second part, we briefly comment on the possible geometrical influence on the determination of the total mass of galaxy clusters. 


\section{SZ effect and the Hubble constant}

Observations of galaxy clusters in the millimetre and $X$-ray wavebands give important information for cosmology. By combining the SZ intensity change and the $X$-ray emission observations, the angular diameter distance to galaxy clusters can be derived. Assuming a cosmological model, this leads to an estimate of the Hubble constant $H_{o}$.

Mauskopf et al. [4] determined $H_{o}$ from $X$-ray measurements of A1835 obtained with ROSAT and from the corresponding millimetric observations of the SZ effect with the Suzie experiment. Assuming an infinitely extended, spherical gas distribution with an isothermal profile $\beta=0.58 \pm 0.02, T_{e o}=9.8_{-1.3}^{+2.3} \mathrm{keV}$, $n_{e o}=5.64_{-1.02}^{+1.61} \times 10^{-2} \mathrm{~cm}^{-3}$, they found $H_{o}=59_{-28}^{+36} \mathrm{~km} \mathrm{~s}^{-1} \mathrm{Mpc}^{-1}$.

Since the hot gas in a real cluster has a finite extension, each of the observed quantities as the Compton parameter $y$ and the $X$-ray surface brightness $S_{x}$ will be smaller than those estimated based on the infinite extension assumption $(l \rightarrow \infty)$. Since the Hubble constant is estimated from the ratio $S_{x} / y^{2}$, in Puy et al. [3] we showed that the relative error $\epsilon_{H_{0}}^{f i n i}$ on the estimate of the Hubble constant, between a spherical distribution with and without finite extension, is given by:

$$
\begin{aligned}
\epsilon_{H_{0}}^{f i n i} & =\frac{H_{0}(\infty)-H_{0}(l)}{H_{0}(\infty)} \\
& =1-\frac{B\left(3 \beta-\frac{1}{2}, \frac{1}{2}\right)\left[B\left(\frac{3}{2} \beta-\frac{1}{2}, \frac{1}{2}\right)-B_{m}\left(\frac{3}{2} \beta-\frac{1}{2}, \frac{1}{2}\right)\right]^{2}}{B^{2}\left(\frac{3}{2} \beta-\frac{1}{2}, \frac{1}{2}\right)\left[B\left(3 \beta-\frac{1}{2}, \frac{1}{2}\right)-B_{m}\left(3 \beta-\frac{1}{2}, \frac{1}{2}\right)\right]}
\end{aligned}
$$

The functions $B$ and $B_{m}$ are combinations of the classical Gamma-functions and the factor $m$ is a cut-off relative to the finite extension (see Puy et al. [3]). In the same way we have analysed the relative error between spherical and aspherical geometries (without finite extension).

Figure 1 shows the influence of geometry and of the assumption of finite extension on the above result using the same input parameters of Mauskopf et al. [4. The left panel shows that for a spherical geometry $H_{o}$ displays a strong dependence on the cluster extension. The right panel gives the value of $H_{o}$ assuming an infinite extended ellipsoid shaped cluster as a function of its axis ratio $\zeta_{1} / \zeta_{3}$.

We see that it is crucial to know the shape of a cluster and its temperature profile. In this context the $X$-ray satellites $X M M$ and Chandra have the necessary spatial and spectral resolution to address this problem on nearby cluster samples.

\section{Morphology of galaxy clusters}

The $\beta$-model [5] is widely used in $X$-ray astronomy to parametrise the gas density profile in clusters of galaxies by fitting their surface brightness profile. In this fitting procedure spherical symmetry is usually assumed, also in cases where the ellipticity of the surface brightness isophotes is manifest. For example Fabricant et al. [6] showed a pronounced ellipticity of the surface brightness for the 


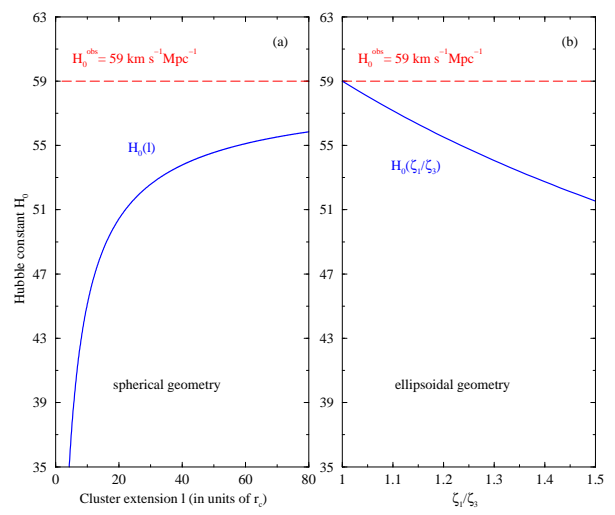

Figure 1: The Hubble constant derived from the data of Mauskopf et al. [4]. Fig. (a) shows the influence of finite extension, while Fig. (b) gives the value of $H_{o}$ assuming an axisymmetric ellipsoidal geometry. In the latter case, oblate or prolate geometry give the same value of $H_{o}$ when taking a line of sight through the cluster center, as is assumed here.

cluster Abell 2256, Allen et al. 7 obtained the same result for the profile of Abell 478 and Neumann \& Böhringer [8] for CL0016+16.

The asphericity of the observed surface brightness let us also ponder on the possible asphericity of the intracluster medium, which can be modelled with an ellipsoidal $\beta$-model rather than with the less accurate spherical one.

Hughes \& Birkinshaw [9] fitted the surface brightness of CL0016+16, which shows an axis ratio of major to minor axis of 1.176, with both circular and elliptical isothermal $\beta$-models obtaining for the best fit parameters $\beta^{\text {circ }}=$ $0.728_{-0.022}^{+0.025}, \sigma_{c}^{\text {circ }}=0.679_{-0.039}^{+0.045}$ arcmin and $\beta^{\text {ell }}=0.737_{-0.022}^{+0.027}, \sigma_{c}^{\text {ell }}=0.746_{-0.044}^{+0.044}$ $\operatorname{arcmin}\left(\sigma_{c}^{\text {ell }}\right.$ is the core radius along the major axis), respectively, with the latter model providing a considerably better fit.

More recently, a Chandra observation of the galaxy cluster RBS797 reveals a pronounced aspherical geometry [10]. The analysis of the image (see Figure 2 ) gives a strong ellipticity, where the axis ratio of major to minor axis varies slightly from 1.3 at a radius of 0.26 arcmin to 1.4 at a radius of 1.7 arcmin (as mentioned in Schindler et al. [10]). Our analysis of the surface brightness profile for RBS797 gives best fit parameters: $\beta^{\text {circ }}=0.62_{-0.03}^{+0.03}, \sigma_{c}^{\text {circ }}=7.32_{-0.7}^{+0.7}$ $\operatorname{arcsec}\left(\right.$ see also [10]) and $\beta^{\text {ell }}=0.59_{-0.02}^{+0.02}, \sigma_{c}^{\text {ell }}=7.89_{-0.9}^{+0.9} \operatorname{arcsec}$ (along the major axis), for the circular and elliptical models respectively [11.

Assuming hydrostatic equilibrium the total mass of a cluster can be estimated from the parameters provided by the surface brightness fit and clearly some care is needed if the galaxy cluster in question shows a pronounced ellipsoidal shape. Assuming hydrostatic equilibrium, the general expression for the total 
mass density $\rho_{\text {tot }}$ is given by

$$
\rho_{t o t}=-\left(\frac{k_{B}}{4 \pi G \mu m_{p}}\right) \vec{\nabla}\left[\frac{1}{\rho_{g}} \vec{\nabla}\left(T_{g} \rho_{g}\right)\right],
$$

where $\rho_{g}$ is the gas mass density, $T_{g}$ its temperature and $\mu m_{p}$ is the mean particle mass of the gas.

In order to obtain the total mass of the cluster, one assumes a spherical geometry and integrates this equation over a sphere with radius $R$. For RBS797 we have additionally investigated the ellipsoidal geometry (for which we assume oblate or prolate shapes) and integrated the equation over an ellipsoid (concentric and similar to the gas core) with major semi-axis $R$ [11]. We thus obtained mass estimates for the spherical shape: $M_{\text {tot }}^{\text {sph }}\left(R=4 \sigma_{c}^{\text {circ }}=29.28 \operatorname{arcsec}\right)=$ $8.66_{-2.3}^{+2.5} \times 10^{13} M_{\odot}$ and $M_{\text {tot }}^{s p h}\left(R=30 \sigma_{c}^{\text {circ }}=219.6\right.$ arcsec $)=6.89_{-1.8}^{+2.0} \times 10^{14} M_{\odot}$ and for ellipsoidal shapes, which are, compared at the same values of $R$, lower than those for the spherical symmetry by $\sim 10 \%$ and $\sim 17 \%$ for oblate or prolate shapes, respectively.

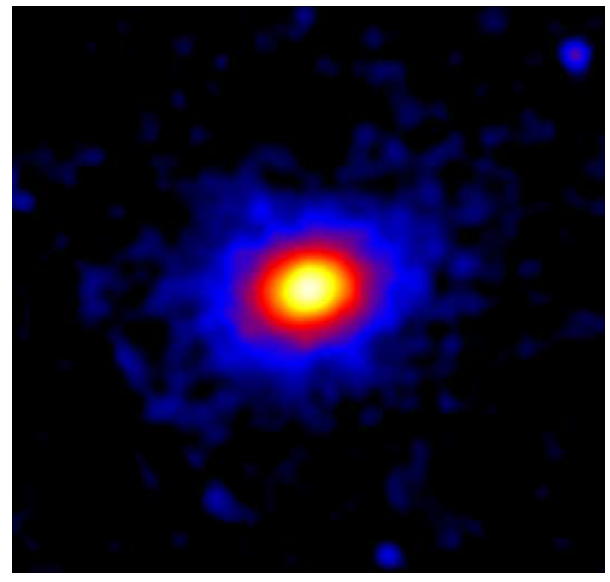

Figure 2: Chandra image of the cluster RBS797. The cluster is rather regular with, however, an ellipticity of 1.3-1.4 in E-W direction. The center and the position angle $\left(\approx-70^{\circ}, \mathrm{N}\right.$ over $\left.\mathrm{E}\right)$ of the various isophotes are almost the same over the entire radius range, from Schindler et al. [10].

\section{Acknowledgments}

We would like to thank Marco de Petris, Massimo Gervasi and Fernanda Luppinacci for organizing 2K1BC Workshop and making it such an enjoyable and stimulating meeting. This work has been supported by the $D^{r}$ Tomalla Foundation and by the Swiss National Science Foundation. 


\section{References}

[1] Cooray, A. R., AA, 339, 623 (1998).

[2] Sulkanen, M., ApJ, 522, 59 (1999).

[3] Puy, D., Grenacher, L., Jetzer, Ph., Signore, M., AA, 363, 415 (2000).

[4] Mauskopf, P., Ade, P., Allen, W. et al. ApJ, 538, 505 (2000).

[5] Cavaliere, A., Fusco-Femiano, R. AA, 49, 137 (1976).

[6] Fabricant, D., Rybicki, G., Gorenstein P., ApJ, 286, 186 (1984).

[7] Allen, S., Fabian, A., Johnstone, D, White, D., Daines, S., Edge, A., Steward, G. MNRAS, 262, 901 (1993).

[8] Neumann, D., Böhringer, H. MNRAS, 289, 123 (1997).

[9] Hughes, J., Birkinshaw M. ApJ, 501, 1 (1998).

[10] Schindler, S., Castillo-Morales, A., De Filippis, E., Schwope, A., Wambsganss, J., Astro-ph/0107504, (2001)

[11] Piffaretti, R., Jetzer, Ph., Puy, D., Schindler, S., in progress (2001). 